\title{
Timing of post-resistance exercise nutrient ingestion: effects on gastric emptying and glucose and amino acid responses in humans
}

\author{
Hideaki Kashima $^{1}$, Kana Sugimura ${ }^{1}$, Kana Taniyawa ${ }^{1}$, Rumi Kondo ${ }^{1}$, Masako Yamaoka Endo ${ }^{1}$, \\ Shota Tanimoto ${ }^{1}$, Toshio Kobayashi ${ }^{2}$, Akira Miura ${ }^{1}$ and Yoshiyuki Fukuba ${ }^{1}{ }^{*}$ \\ ${ }^{1}$ Department of Exercise Science and Physiology, School of Health Sciences, Prefectural University of Hiroshima, 1-1-71 \\ Ujina-bigashi, Minami-ku, Hiroshima 734-8558, Japan \\ ${ }^{2}$ Department of Health Promotion and Development, Graduate School of Health Sciences, Hiroshima University, 1-2-3 Kasumi \\ Minami-ku, Hiroshima 734-8551, Japan
}

(Submitted 15 February 2018 - Final revision received 22 May 2018 - Accepted 1 August 2018 - First published online 17 September 2018)

\section{Abstract}

This study examined the effects of post-resistance exercise protein ingestion timing on the rate of gastric emptying (GE) and blood glucose (BG) and plasma branched-chain amino acid (BCAA) responses. In all, eleven healthy participants randomly ingested $400 \mathrm{ml}$ of a nutrient-rich drink containing $12 \mathrm{~g}$ carbohydrates and $20 \mathrm{~g}$ protein at rest (Con), at $5 \mathrm{~min}$ (post-exercise (PE)-5) or at 30 min (PE-30) after a single bout of strenuous resistance exercises. The first and second sets comprised ten repetitions at $50 \%$ of each participant's one-repetition maximum (1RM). The third, fourth and fifth sets comprised ten repetitions at $75 \%$ of $1 \mathrm{RM}$, and the sixth set involved repeated repetitions until exhaustion. Following ingestion of the nutrient-rich drink, we assessed the GE rate using ${ }^{13} \mathrm{C}$-sodium acetate breath test and evaluated two parameters according to the $T_{\text {max-calc }}$ (time when the recovery per hour is maximised), which is a standard analytical method, and $T_{1 / 2}$ (time when the total cumulative dose of $\left[{ }^{13} \mathrm{CO}_{2}\right]$ reaches one-half). $T_{\text {max-calc }}$ and $T_{1 / 2}$ were slower for the PE- 5 condition than for either the PE-30 or Con condition ( $T_{\text {max-calc }}$ Con: 53 (sD 7) min, PE-5: 83 (sD 16) min, PE-30: 62 (sD 9) min, $T_{1 / 2}$; Con: 91 (sD 7) min, PE-5: 113 (sD 21) min, PE-30: 91 (sD 11) min, $P<0 \cdot 05$ ). BG and BCAA responses were also slower for the PE- 5 condition than for either the PE-30 or Con condition. Ingesting nutrients immediately after strenuous resistance exercise acutely delayed GE, which affected BG and plasma BCAA levels in blood circulation.

Key words: Blood glucose: Gastric emptying rate: Gastrointestinal disorders: Plasma amino acids: Strenuous resistance exercise

According to a new position paper, carbohydrate-protein supplementation during the post-exercise (PE) recovery period promotes muscle protein anabolism ${ }^{(1)}$. To achieve maximum muscle adaptation to exercise, laboratory-based nutritional studies have reported that 'immediate' PE consumption of protein can elicit greater muscle protein synthesis, whereas fasting for 2-3 h after exercise blunts this response $\mathrm{e}^{(2)}$. Therefore, carbohydrate-protein supplementation as soon as possible (at least within $45 \mathrm{~min}$ ) after endurance exercise has been recommended; these recommendations are also applicable to resistance exercise ${ }^{(3)}$.

However, during and immediately after exercise, $25-70 \%$ of endurance athletes experience transient anorexia as well as gastrointestinal (GI) symptoms, such as nausea and stomach pain $^{(4-6)}$. These exercise-induced GI symptoms can occur frequently, thereby decreasing exercise performance and subsequent recovery in athletes. Although there are numerous causes of exercise-induced GI symptoms, some recent studies considered that reduction of GI blood flow (BF) is a likely contributor $^{(6-8)}$. Strenuous exercise of high intensity and/or prolonged duration often reduces BF in the celiac artery $(\mathrm{CA})^{(9)}$, superior mesenteric artery $(\mathrm{SMA})^{(9,10)}$, gastric mucosa ${ }^{(11-13)}$, portal vein $^{(14)}$ and whole viscera ${ }^{(15,16)}$, potentially damaging the small intestine mucosal cells ${ }^{(13,17,18)}$. When the GI tract is hypoperfused following exercise, the consumption of a nutrient-rich beverage may impair digestion and/or absorption. In this regard, van Wijck et al. ${ }^{(17)}$ observed the appearance of plasma phenylalanine after ingestion of $20 \mathrm{~g}$ dietary protein immediately after high-intensity resistance exercise. Compared with a no exercise trial, plasma phenylalanine levels lowered for approximately 30-60 min during the PE phase. The authors interpreted that strenuous exercise-induced GI hypoperfusion damaged intestinal mucosal cells and consequently decreased plasma phenylalanine appearance, thereby impairing GI function, although GI BF and its associated functions were not directly measured.

Abbreviations: 1RM, one-repetition maximum; BCAA, branched-chain amino acids; BF, blood flow; bpm, beats per min; CA, celiac artery; Con, control trial; GE, gastric emptying; GI, gastrointestinal; HR, heart rate; PE, post-exercise; SMA, superior mesenteric artery.

* Corresponding author: Y. Fukuba, fax +81 822519806 , email fukuba@pu-hiroshima.ac.jp 
To elucidate the relationship between GI function and BF, we simultaneously measured and evaluated the rate of gastric emptying (GE), CA and SMA BF after $30 \mathrm{~min}$ high-intensity intermittent leg cycling exercise comprising a $120 \% \dot{\mathrm{V}} \mathrm{O}_{2}$ peak for $20 \mathrm{~s}$ followed by $20 \mathrm{~W}$ for $40 \mathrm{~s}^{(18)}$. The CA supplies blood to the stomach, pancreas, spleen and liver, and the SMA primarily supplies blood to the jejunum and ileum. The participants ingested a carbohydrate-protein drink either 5 min (i.e. concurrently with decreased $\mathrm{CA} \mathrm{BF}$ ) or $30 \mathrm{~min}$ (i.e. as $\mathrm{CA} \mathrm{BF}$ is recovering) $\mathrm{PE}$. In the $5 \mathrm{~min} \mathrm{PE}$ trial, the GE rate and SMA $\mathrm{BF}$ responses after ingestion of the carbohydrate-protein drink were slower than those observed in the $30 \mathrm{~min}$ PE trial. These results suggested that PE GI hypoperfusion acutely suppressed subsequent digestive and/or absorptive functions. As this study used a laboratory-based basic science approach, our data suggest that athletes and training enthusiasts should avoid carbohydrate-protein supplementation immediately after strenuous exercise.

Given the range of competitive sports and fitness activities, it is meaningful to examine GI effects related to timing of nutrient supplementation after exercise. Several previous reviews have summarised the effects of exercise intensity and duration on the rate of GE during 'endurance exercise ${ }^{(19-22)}$; however, there is very limited evidence on the effects of GE rate after exercise ${ }^{(18,23)}$. Surprisingly, very little is known about the rate of GE during and after 'resistance exercise', as indicated by a recent review $^{(20)}$, despite the importance of promoting quick rehydration, refuelling and repair of damaged tissues following exercise $^{(4,24,25)}$. Therefore, to understand GE after resistance exercise, we investigated the effects of the timing of carbohydrateprotein supplementation on GE rate after strenuous resistance exercise. The protocol used was similar to that of our previous study to compare the responses between two different types of exercises. In addition, we examined plasma branched-chain amino acids (BCAA) and blood glucose responses, because our previous study failed to evaluate the appearance of nutrients into blood circulation after ingestion of a nutrient-rich drink ${ }^{(18)}$. Thus, the manner in which the acute alternative of GE rate (i.e. partial digestive process) could also impact the subsequent digestive-absorptive rates in the small intestines during the PE phase has not been clarified. We demonstrated that GE was suppressed nearly immediately (i.e. at $5 \mathrm{~min}$, but not $30 \mathrm{~min}$ ) after strenuous resistance exercise, which consequently delayed plasma BCAA and blood glucose appearance into the blood circulation.

\section{Methods \\ Participants}

In all, eleven healthy young subjects (seven females and four males; age, 21 (SD 3) years; height, 162 (SD 8) cm; weight, 56 (SD 5) kg; BMI, 22 (sD 2) kg/m²) participated in this study. The participants were young, healthy and normotensive. No participants smoked or took any medication, and none had any histories of autonomic dysfunction, as well as cardiovascular or GI tract disorders. The study protocol was performed in compliance with the Declaration of Helsinki, and the Ethics Committee of the Prefectural University of Hiroshima approved the study (approval no. HH007). Before study commencement, each participant provided written informed consent for participation.

\section{Preliminary test session}

As a baseline/pre-test, we determined the one-repetition maximum (1RM) for each of three resistance exercises (lat-pulldown, chest-press and leg-presses) using the training machines (Technogym-Element Plus Line; Technogym Co. Ltd). We defined the 1RM as the maximum amount of weight each participant could lift during one repetition. This baseline/pre-test was performed on a different day from the main protocols, as described in the forthcoming text.

\section{Main trial session}

Each participant performed the following three protocols in random order. Female participants participated in each protocol during the same phase of their menstrual cycles, because menstruation affects GE and blood glucose, insulin and glucagon-like peptide- 1 concentrations ${ }^{(26)}$. Males participated a maximum of once per week. An overview of the exercise protocol is shown in Fig. 1. Participants arrived at approximately 08.30-10.30 hours in the laboratory, having abstained from strenuous exercise, alcohol and caffeine ingestion for at least $1 \mathrm{~d}$. Each participant started three protocols during the same hour. In the $3 \mathrm{~h}$ before each

\section{Non-exercise}
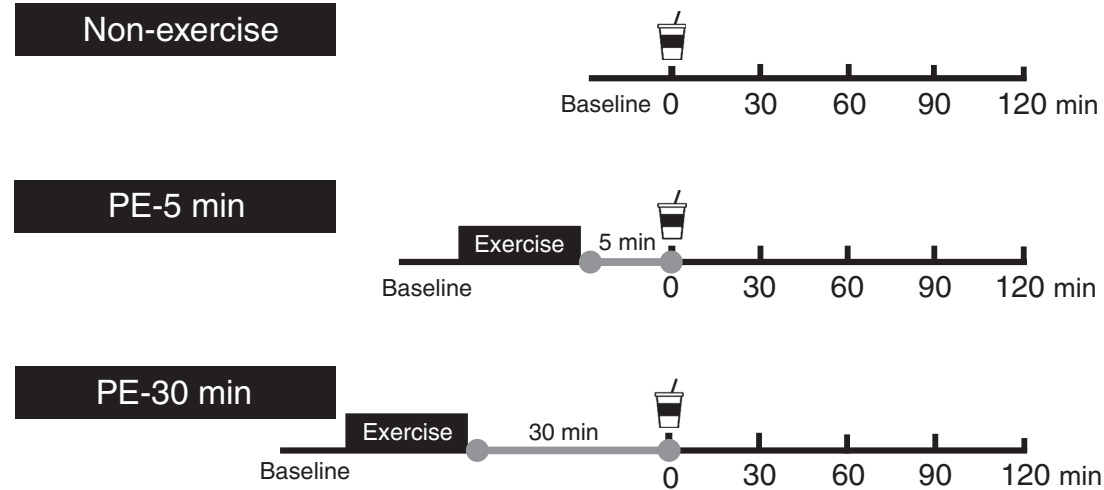

Fig. 1. Schematic representation of the study protocol. Participants ingested the carbohydrate-protein drink either at 5 min (post-exercise (PE)-5) or 30 min (PE-30) after strenuous resistance exercise and then rested for $120 \mathrm{~min}$. In the Con, participants ingested the same nutrient-rich drink without engaging in exercise. 
experimental trial, participants consumed a semi-liquid standardised meal of approximately $836.8 \mathrm{~kJ}$ (200 kcal) (SAVAS Energy Maker Jelly; Meiji) comprising $\mathrm{CHO}(49.7 \mathrm{~g})$, protein $(0 \mathrm{~g})$ and fat $(0 \mathrm{~g})$. Using ultrasonography, we confirmed that the diet had completely emptied from the stomach at experiment onset ${ }^{(18)}$. Participants were seated in a chair in a quiet resting room for 5 min (baseline measurement), where the temperature and humidity were maintained at $24 \pm 1^{\circ} \mathrm{C}$ and $46 \pm 4 \%$, respectively. After this, the participants moved to the exercise room to perform resistance exercises for approximately $30 \mathrm{~min}$, where the temperature and humidity were maintained at $26 \pm 2{ }^{\circ} \mathrm{C}$ and $66 \pm 9 \%$, respectively. Following warm-up (i.e. standardised dynamic stretching) for 2 min, a single bout of resistance exercise was performed, consisting of six sets on a circuit of abdominal curls, lat-pull-downs, chest-presses and leg-presses. During the first and second sets, the exercise intensity and volume were set at 50\% of 1RM and ten repetitions. During the third, fourth and fifth sets, the intensity and volume were set at $75 \%$ of $1 \mathrm{RM}$ and ten repetitions. In the final (sixth) set, the intensity and volume were set at $75 \%$ of $1 \mathrm{RM}$ and the participants repeated each exercise until exhaustion. During those exercises, duration of each concentric and eccentric action was controlled at 1.5-2 s using an electronic metronome. During the first and second sets, ten abdominal curl repetitions were performed on a flat floor with the hips and knees flexed to $90^{\circ}$ and legs supported on a platform. The participant's hands were placed behind his or her head and the participant's shoulders were elevated from the flat floor. Participants sat up until their elbows touched their knees, leaving the trunk approximately 75 $90^{\circ}$ from the floor. During the third, fourth, fifth and sixth sets, the participant's trunk was inclined backward at a $10^{\circ}$ angle from the flat floor. During the third, fourth and fifth sets, ten abdominal curls were performed, and in the final (sixth) set, each participant completed maximal voluntary repetitions for $40 \mathrm{~s}$. During abdominal curls in the first through fifth sets, the duration of each concentric and eccentric action was controlled at $1 \cdot 5-2 \mathrm{~s}$ using an electronic metronome. Following the resistance exercises, each participant was seated in a chair to rest. During the PE period, each participant ingested $400 \mathrm{ml}$ of nutrient-rich drink containing $12 \mathrm{~g}$ carbohydrate and $20 \mathrm{~g}$ whey protein (ZAVAS Protein Drink; Meiji Co. Ltd) at either $5 \mathrm{~min}$ (PE-5) or $30 \mathrm{~min}$ (PE-30) and then rested for $120 \mathrm{~min}$. As a control trial (Con), each participant ingested the same nutrientrich drink without exercise immediately after baseline measurement. Participants were instructed to consume the nutrientrich drink for $2 \mathrm{~min}$. Each participant performed the three protocols in random order. At first, participants were allocated to the Con $(n$ 4), PE-5 ( $n$ 4) and PE-30 ( $n$ 3).

\section{Measurements}

With the exception of during and 5 min after exercise, heart rate (HR) was continuously monitored using an electrocardiogram (Colin BP-88S; Colin Co. Ltd).

GE rate was evaluated using the ${ }^{13} \mathrm{C}$-sodium acetate breath test ${ }^{(27)}$. After oral administration, ${ }^{13} \mathrm{C}$-sodium acetate is absorbed rapidly in the small intestine (but not in the stomach), metabolised and finally expired as $\left[{ }^{13} \mathrm{CO}_{2}\right]$. This breath test is a widely-used, reproducible and non-invasive alternative to scintigraphy (which has the drawback of radiation exposure $)^{(27)}$. We dissolved $100 \mathrm{mg}$ of ${ }^{13} \mathrm{C}$-sodium acetate into the nutrient-rich drink. Participants were instructed to hold their breath for $10 \mathrm{~s}$ to empty end-expiratory breath in a sample foil bag. Baseline breath samples were collected using a largecapacity bag (PAYLORI-BAG5 L; Fukuda Denshi). After ingestion of the nutrient-rich drink, breath samples were collected using a small-capacity bag (PAYLORI-BAG20; Fukuda Denshi) at 5- and 10-min intervals for 5-60 and 70-120 s, respectively. $\left[{ }^{13} \mathrm{CO}_{2}\right]$ enrichment in breath was measured using an isotope ratio mass spectrometer (POCone; Otsuka Electronics). $\mathrm{CO}_{2}$ production per body surface area was assumed to be $300 \mathrm{mmol} /$ $\mathrm{m}^{2}$ per $\mathrm{h}$. The body surface area was calculated according to a weight-height formula ${ }^{(28)}$. We determined longitudinal measures of the percentage $\left[{ }^{13} \mathrm{CO}_{2}\right]$ recovery per hour and the cumulative percentage of $\left[{ }^{13} \mathrm{CO}_{2}\right]$ recovery. The times when the $\left[{ }^{13} \mathrm{CO}_{2}\right]$ recovery per hour reached a maximum (i.e. $T_{\text {max-calc }}$ ) and when the total cumulative dose of the $\left[{ }^{13} \mathrm{CO}_{2}\right]$ reached onehalf (i.e. $T_{1 / 2}$ ) were calculated according to a standard analytical $\operatorname{method}^{(27)}$. These parameters are closely correlated with GE using scintigraphy ${ }^{(27,29,30)}$ and the Wagner-Nelson method ${ }^{(31)}$.

\section{Blood sampling}

Capillary blood samples were collected via left index and middle finger skin pricks before exercise (baseline), immediately after exercise, just before and at 15, 30, 45, 60, 90 and $120 \mathrm{~min}$ after ingestion of the nutrient-rich drink. Blood glucose and lactate concentrations were analysed by dedicated measurement devices (Glucocard Diameter-alpha GT-1661 and Lactate Pro2 LT-1730; Arkray). Blood samples were collected into post-heparinised $75-\mu$ l capillary tubes and centrifuged at $3000 \boldsymbol{g}$ for $5 \mathrm{~min}$ at room temperature to obtain plasma samples. The plasma samples were deproteinised with acetonitrile and centrifuged at $15000 \mathrm{~g}$ and $4^{\circ} \mathrm{C}$ for $5 \mathrm{~min}$. Then, $95 \mu \mathrm{l}$ of deproteinised supernatants samples were mixed with $5 \mu$ of norleucine as an internal standard solution and then those samples were stored at $-80^{\circ} \mathrm{C}$ before analysis. The mixtures were derivatised using the AccQTag Ultra Deriviatisation Kit (Waters). Plasma BCAA concentrations, which are expressed as the total concentrations of valine, isoleucine and leucine in plasma, were determined using the Waters ACQUITY Ultra Performance LC System equipped with an AccQTag Ultra Column $(2 \cdot 1 \times 100 \mathrm{~mm}$; Waters) and TUV detector (Waters). Blood glucose values and plasma BCAA concentrations were calculated as absolute change from baseline. BCAA responses were calculated as the incremental AUC (iAUC) above baseline values, after ingestion of the nutrient-rich drink. The intersample variability and reproducibility in BCAA values when comparing venous with capillary blood were acceptable (most $<10$ and $20 \%$, respectively $)^{(32)}$.

\section{Participants' average appetite score}

We assessed participants' GI symptoms and motivation to eat using 100-mm visual analogue scales before exercise, immediately after exercise and every $30 \mathrm{~min}$ thereafter. The GI symptoms of interest included distention, nausea and abdominal 
pain. All scales ranged from $0 \mathrm{~mm}$ (feel nothing at all) to $100 \mathrm{~mm}$ (very strong feeling). Each participant's motivation to eat was assessed by measuring hunger, fullness, desire to eat and prospective consumption. The scales for hunger, desire to eat and prospective consumption ranged from $0 \mathrm{~mm}$ (no hunger, desire to eat or food consumption) to $100 \mathrm{~mm}$ (very hungry, strong desire to eat or consume food). For fullness, the scale ranged from $0 \mathrm{~mm}$ (not full at all) to $100 \mathrm{~mm}$ (very full). Using the scores of these four questions, each participant's average appetite score was calculated for each measurement time point according to the following formula ${ }^{(33,34)}$ :

Participants average appetite score $(\mathrm{mm})$

$=($ desire to eat + hunger $+(100-$ fullness $)$

+ prospective consumption) $/ 4$.

\section{Power and statistical analysis}

In a pilot experiment (four participants; two females and two males), we compared $T_{\text {max-calc }}$ (i.e. main GE rate index) during conditions of non-exercise (i.e. Con) and 5 or $30 \mathrm{~min}$ postresistance exercise (i.e. $\mathrm{PE}-5$ and $\mathrm{PE}-30$, respectively). The mean values and standard errors of $T_{\text {max-calc }}$ for Con, PE- 5 and PE-30 were 53.7 (SD 5.5), 80.9 (SD 19.1) and 63.5 (SD 6.5) min, respectively. Then, we ran a statistical power analysis based on this outcome using G*Power (version 3.1.9.2) with four participants per condition. We obtained an effect size of 1.435 for a critical $F_{2,6}$ of 5.143 at $\alpha$ error probability of 0.05 and power ( $1-\beta$ error probability) of $0 \cdot 80$. The calculated total sample size was nine and, consequently, we planned to recruit a total of eleven participants to allow for dropouts.

Data are expressed as means and standard deviations of means. The effects of time and treatment on HR, blood lactate concentrations, blood glucose and plasma BCAA concentrations and participant's GI compliance and appetite scores were tested by two-way repeated ANOVA. When a significant effect was detected, Dunnett's and Tukey's post hoc tests were conducted to reveal the effects of time (change from baseline) and treatments, respectively. The effect of treatment on $T_{\text {max-calc }}$ and $T_{1 / 2}$ and iAUC for BCAA were tested by one-way repeated ANOVA. When a significant effect was detected, Tukey's post hoc tests were conducted. The relationship between blood lactate concentration and $T_{\text {max-calc }}$ was evaluated by Pearson's correlation coefficient. The two-sided statistical significance level was set at $P \leq 0 \cdot 05$. All statistical analyses were performed with SPSS PASW 18 statistics software (SPSS Inc.).

\section{Results}

The mean pre-test 1RM for lat-pull-down, chest-press and legpresses were 55 (sD 20), 49 (sD 25) and 153 (sD 46) kg, respectively.

\section{Central circulatory responses and blood lactate concentration}

Baseline HR values did not significantly differ among trials (Con: 66 (sD 9) beats per min (bpm), PE-5: 67 (SD 11) bpm, PE-30: 63 (sD 11) bpm) (Fig. 2(a)). During the PE-5 and PE-30 conditions, there were no statistically significant between-group differences in HR immediately after exercise (PE-5: 99 (SD 13) bpm, PE-30: 98 (sD 11) bpm) ( $P>0 \cdot 05)$. During the postingestion phase, HR values were the highest during the PE-5 condition $(P<0.05)$. The values were higher during the PE-30 condition than during the Con condition $(P<0.05)$.

Baseline blood lactate concentrations did not significantly vary among the trials (Con: $1 \cdot 1(\mathrm{sD} 0 \cdot 3) \mathrm{mmol} / \mathrm{l}, \mathrm{PE}-5: 1 \cdot 2$ (SD 0.3) $\mathrm{mmol} / \mathrm{l}$, PE-30: $1.0(\mathrm{sD} 0.3) \mathrm{mmol} / \mathrm{l}$ ) (Fig. 2(b)). During the PE- 5 and PE-30 conditions, there were no statistically significant between-group differences in blood lactate concentrations immediately after exercise (PE-5: 16.4 (sD 3.2) mmol/1, PE-30: $15 \cdot 8$ (sD 3.0$) \mathrm{mmol} / \mathrm{l})(P>0.05)$. Blood lactate concentrations just before ingestion of the nutrient-rich drink were higher during the PE-5 than during the PE-30 condition (PE-5: 15.0 (SD 4.1) $\mathrm{mmol} / \mathrm{l}$, PE-30: 6.9 (sD 2.4) $\mathrm{mmol} / \mathrm{l}, P<0 \cdot 05$.).

\section{Gastric emptying rate}

Longitudinal pulmonary $\left[{ }^{13} \mathrm{CO}_{2}\right]$ excretion rates are shown in Fig. 3(a). $T_{\text {max-calc }}$ for PE-5 showed a significant delay compared with the Con and PE-30 conditions (Con: 53 (SD 7) min, PE-5: 83 (sD 16) min, PE-30: 62 (sD 9) min, $P<0 \cdot 05$, Fig. 3(b)). $T_{1 / 2}$ for PE-5 also showed a significant delay compared with the Con and PE-30 conditions (Con: 91 (sD 7) min, PE-5: 113 (sD 21) min, PE-30: 91 (sD 11) min, $P<0 \cdot 05$, Fig. 3(c)). For PE-30, $T_{\text {max-calc }}$ and $T_{1 / 2}$ did not significantly differ from the Con condition. $T_{\text {max-calc }}$ was significantly correlated with blood lactate concentration just before ingestion of the nutrient-rich drink $\left(R^{2} 0.62, P<0.05\right)$ (Fig. 3(d)).

\section{Blood glucose and plasma branched-amino acids responses}

Longitudinal blood glucose and plasma amino acid responses are shown in Figs. 4 and 5, respectively. After ingestion of the nutrient-rich drink, the blood glucose concentration for the PE-5 condition was significantly increased from baseline at $30 \mathrm{~min}$ $(P<0.05)$, whereas the blood glucose concentrations for the Con and PE-30 conditions were significantly increased at $15 \mathrm{~min}$ $(P<0 \cdot 05)$. At $60-90 \mathrm{~min}$, the blood glucose concentration was higher for the PE- 5 condition than for the Con $(P<0 \cdot 05)$. During the post-ingestion phase, plasma valine concentrations were significantly increased from baseline at 30-120, 60-120 and 45120 min during the Con, PE- 5 and PE-30 conditions, respectively $(P<0.05)$. Plasma valine concentrations observed during the PE-5 and PE-30 conditions were lower than those observed during the Con condition at $30 \mathrm{~min}(P<0.05)$. The plasma isoleucine concentrations were significantly increased from baseline at 30-120, 45-120 and 30-120 min during the Con, PE-5 and PE-30 conditions, respectively $(P<0 \cdot 05)$. Plasma isoleucine concentrations during the PE- 5 condition were lower than those during the Con condition at 15-45 min and the PE-30 condition at $15 \mathrm{~min}(P<0 \cdot 05)$. Plasma isoleucine concentrations during the PE-30 condition were lower than those during the Con condition at $30 \mathrm{~min}(P<0.05)$. Plasma isoleucine concentrations during the PE- 5 condition were higher than those during the PE-30 conditions at $120 \mathrm{~min}(P<0.05)$. The plasma leucine concentrations were significantly increased from baseline at 
(a)
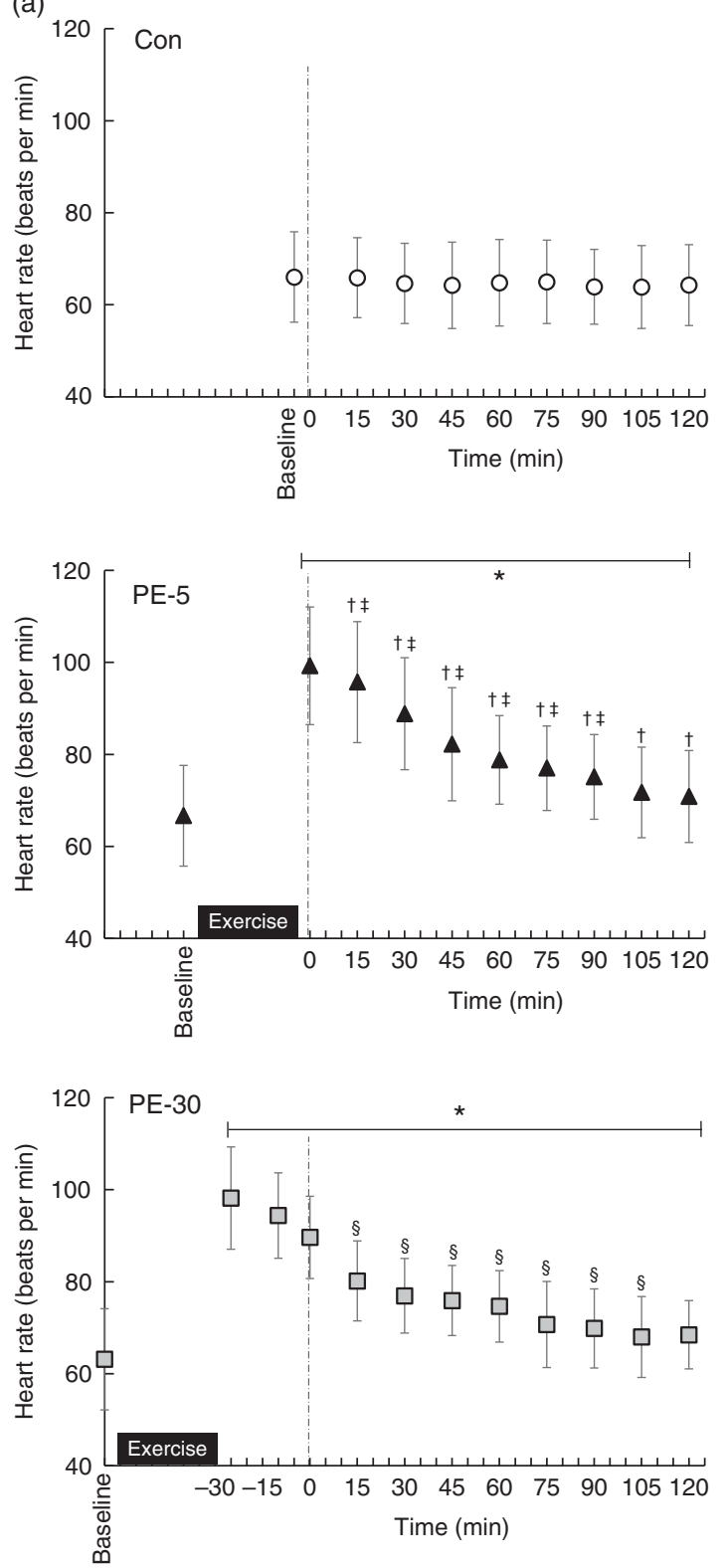

(b)
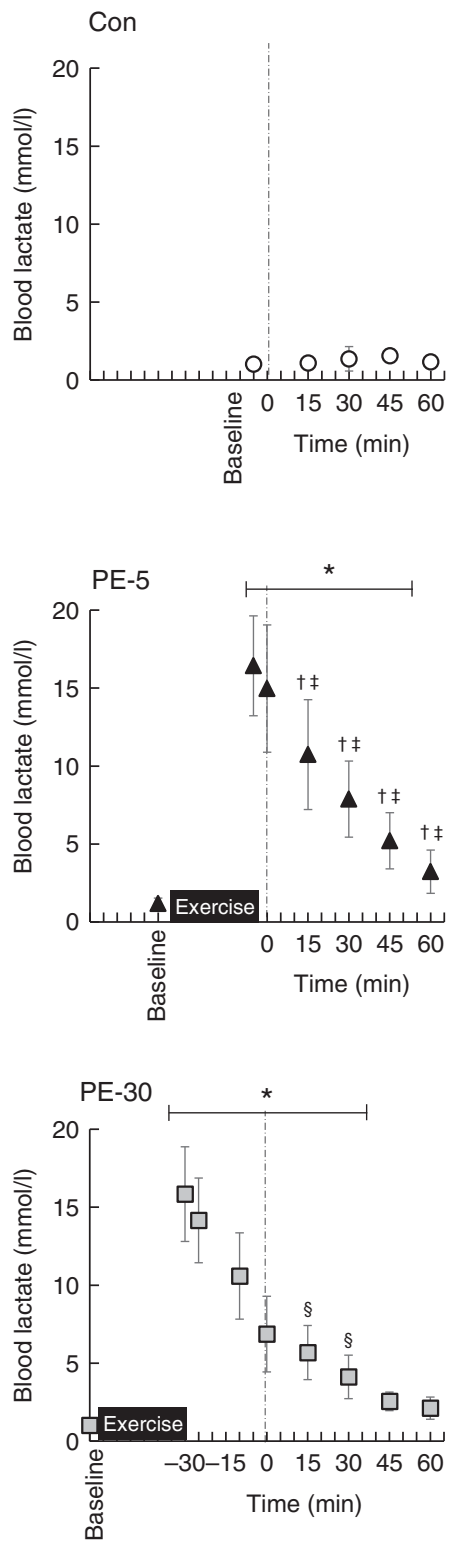

Fig. 2. Longitudinal heart rate (a) and blood lactate (b) responses, except for those obtained during exercise. The upper, middle and lower panels indicate no exercise (Con), $5 \mathrm{~min}$ post-exercise (PE-5) and $30 \mathrm{~min}$ post-exercise (PE-30), respectively. At $0 \mathrm{~min}$, the vertical dotted line denotes the timing of carbohydrate-protein supplementation. Heart rate and blood lactate levels were tested by two-way repeated ANOVA. When a significant difference was detected, we conducted Dunnett's and Tukey's post hoc tests to determine the effects of time (the change from baseline) and treatments, respectively. Values are means, with standard deviations represented by vertical bars. * Mean value was significantly different ( $v$. baseline; $P<0.05)$; $†$ Mean value was significantly different for $P E-5 v$. Con $(P<0.05)$. $\ddagger$ Mean value was significantly different for PE-5 v. PE-30 $(P<0.05)$. § Mean value was significantly different for Con $v$. PE-30 $(P<0.05)$.

30-120, 45-120 and 30-120 min during the Con, PE-5 and PE-30 conditions, respectively $(P<0 \cdot 05)$. Plasma leucine concentrations observed during the PE- 5 condition were lower than those observed during the Con condition at $15-60 \mathrm{~min}$ and the PE-30 condition at $15 \mathrm{~min}(P<0 \cdot 05)$. Plasma leucine concentrations observed during the PE-30 conditions were lower than those observed during the Con condition at $30 \mathrm{~min}(P<0 \cdot 05)$. Plasma leucine concentrations during the PE- 5 condition were higher than those during the PE-30 condition at $120 \mathrm{~min}(P<0.05)$. The iAUC of BCAA were significantly lower during the PE- 5 condition compared with the Con condition $(P<0 \cdot 05)$. During the
PE-30 condition, the AUC of BCAA were statistically equivalent to those observed during the Con condition. This difference almost reached significance compared with the PE- 5 condition $(P=0.08)$ (Con: 45291 (sD 18575) nmol $/ \mathrm{ml}, \mathrm{PE}-5$ : 26352 (sD 19 445) nmol/ml, PE-30: 39895 (sD 9685$) \mathrm{nmol} / \mathrm{ml}$ ).

\section{Participants' gastrointestinal disorder and appetite scores}

During baseline/pre-testing, no participants complained of GI symptoms during any of the trials. During the PE- 5 and PE-30 conditions, stomach distension, stomach pain and nausea 
(a)

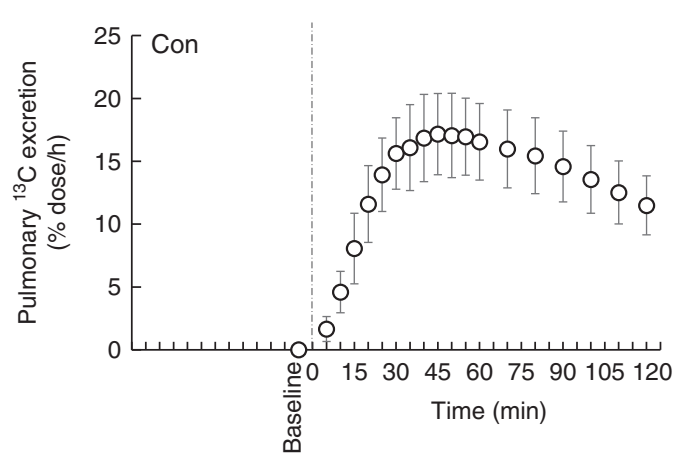

(b)

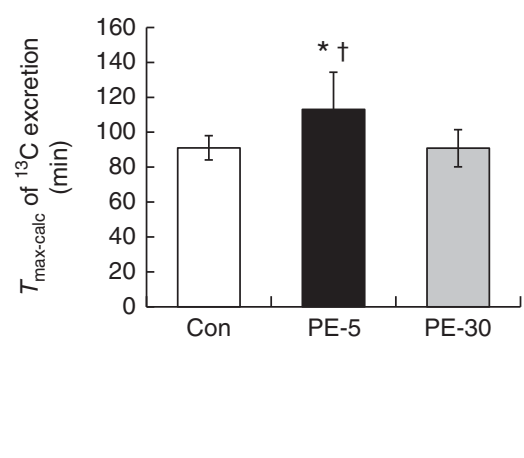

(c)
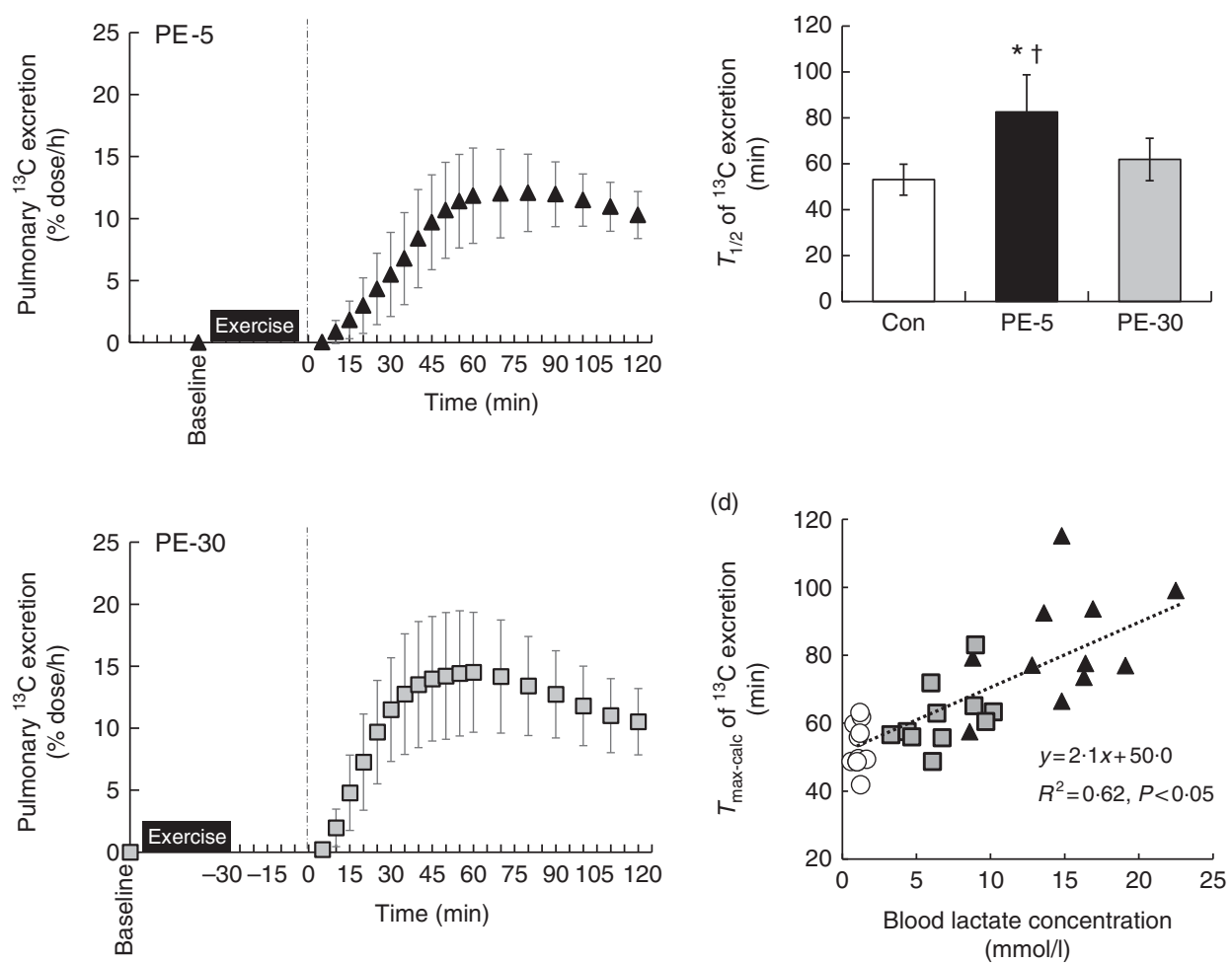

Fig. 3. Gastric emptying rate using the ${ }^{13} \mathrm{C}$ breath test. Longitudinal ${ }^{13} \mathrm{C}$ excretion responses (a) after ingestion of nutrient drink. The upper, middle and lower panels indicate no exercise (Con), $5 \mathrm{~min}$ post-exercise (PE-5) and $30 \mathrm{~min}$ post-exercise (PE-30), respectively. At 0 min, the vertical dotted line denotes the timing of carbohydrate-protein supplementation. The effect of treatment on $T_{\text {max-calc }}(\mathrm{b})$ and $T_{1 / 2}$ (c) were tested by one-way repeated ANOVA. When a significant difference was detected, we conducted Tukey's post hoc test. $T_{\text {max-calc }}$ and $T_{1 / 2}$ of ${ }^{13} \mathrm{C}$ excretion rate, which are the gastric emptying indices, were altered by timing of postexercise nutrient supplementation. The relationship between blood lactate concentration and $T_{\text {max-calc }}$ was evaluated by Pearson's correlation coefficient (d). $T_{\text {max-calc }}$ was significantly correlated with blood lactate concentrations just before ingestion of the nutrient-rich drink. The white circle, black triangle and grey square represent Con, PE-5 and PE-30, respectively. Values are means, with standard deviations represented by vertical bars. * Mean value was significantly different for PE-5 $v$. Con $(P<0.05)$. † Mean value was significantly different for PE-5 v. PE-30 $(P<0.05)$.

scores exhibited no statistically significant differences after exercise and ingestion of the nutrient-rich drink (Table 1). Nausea scores were higher during the PE- 5 condition than the Con condition at $30 \mathrm{~min}(P<0.05)$. Although we could not measure from 5-25 min after ingestion, during the PE-5 condition, four participants complained of severe nausea at 5-30 min after ingestion of the nutrient-rich drink.

Baseline average appetite scores did not differ among the three conditions (Con: 77 (SD 16) mm, PE-5: 78 (SD 15) mm, PE-30: 83 (sD 15) mm). During the PE-5 and PE-30 conditions, average appetite scores immediately PE demonstrated equivalent decreases from baseline (PE-5: 33 (SD 11) mm, PE-30: 29 (sD 13) $\mathrm{mm}, P<0 \cdot 05$ ). At $30 \mathrm{~min}$ after ingestion of the nutrientrich drink, average appetite scores were lower during the PE-5 condition than either the Con or PE-30 condition $(P<0.05)$.

\section{Discussion}

We compared GE rate and BCAA and blood glucose responses during conditions of non-exercise (i.e. Con) and 5 or $30 \mathrm{~min}$ 

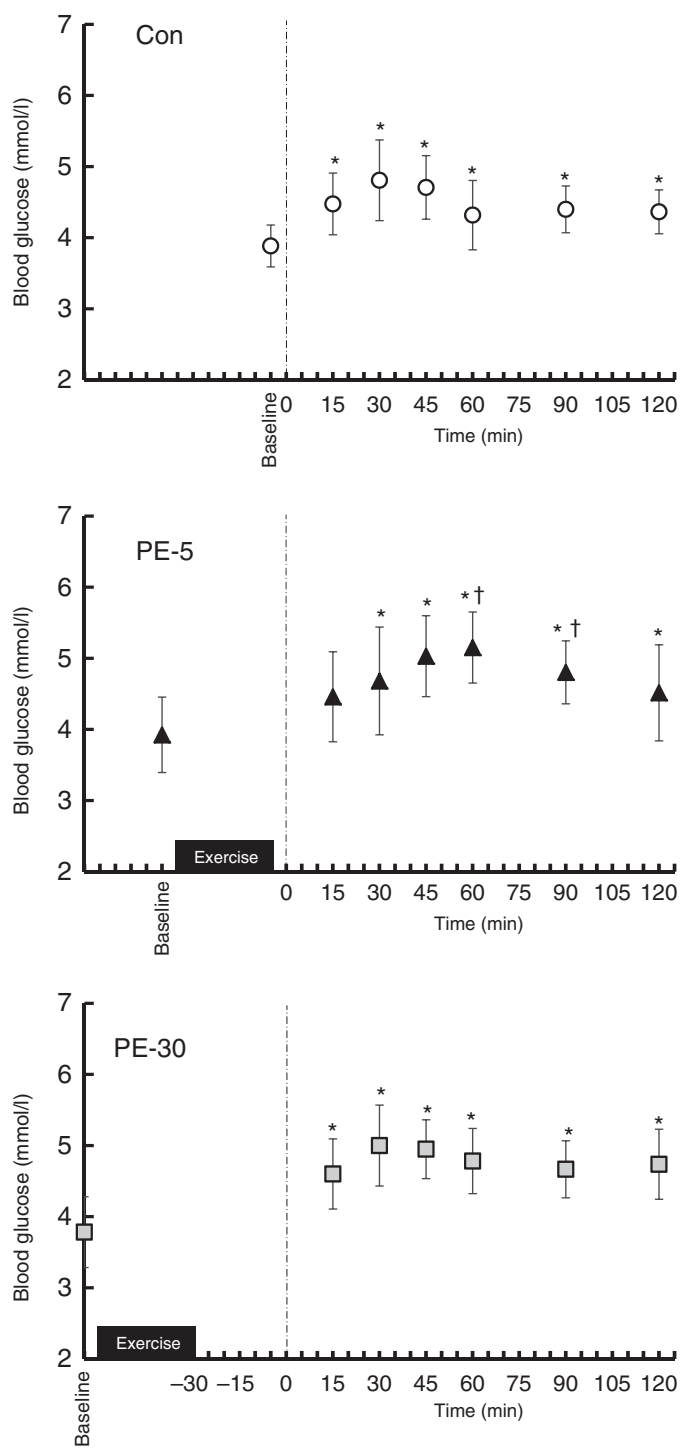

Fig. 4. Longitudinal blood glucose responses, except for those during exercise. The upper, middle and lower panels indicate no exercise (Con), $5 \mathrm{~min}$ postexercise (PE-5) and $30 \mathrm{~min}$ post-exercise (PE-30), respectively. At $0 \mathrm{~min}$, the vertical dotted line denotes the timing of carbohydrate-protein supplementation. Blood glucose responses were tested by two-way repeated ANOVA. When a significant difference was detected, we conducted Dunnett's and Tukey's post hoc tests to determine the effects of time (the change from baseline) and treatments, respectively. Values are means, with standard deviations represented by vertical bars. * Mean value was significantly different ( $v$. baseline; $P<0.05)$. † Mean value was significantly different for PE-5 v. Con $(P<0.05)$.

post-resistance exercise (i.e. PE-5 and PE-30, respectively) to elucidate the impact of timing of nutrient ingestion on digestion-absorption rates. During the PE- 5 condition, GE rate was delayed compared with the Con and PE-30 conditions, resulting in slower BCAA and blood glucose responses, compared with the Con or PE-30 conditions. During the PE-30 condition, there were no statistically significant differences from the Con condition for GE rate and BCAA and blood glucose responses. The rate of $\mathrm{GE}$ was altered by the timing of nutrient consumption after strenuous resistance exercise. This phenomenon might be the key to determining subsequent digestive-absorptive rates.
The major finding in the present study was that GE was strongly suppressed when the nutrient-rich drink was given immediately after strenuous resistance exercise (i.e. PE-5). In contrast, when the nutrient drink was ingested $30 \mathrm{~min}$ after strenuous resistance exercise (i.e. PE-30), GE was unaffected. These results agree with the findings of Evans et al. ${ }^{(23)}$, our previous human study ${ }^{(18)}$, and the animal-based findings of Silva et al. ${ }^{(35)}$. Evans et al. ${ }^{(23)}$ reported that GE rates following the ingestion of a carbohydrate beverage $(595 \mathrm{ml}$ of $5 \%$ glucose solution) at $30 \mathrm{~min}$ after high-intensity interval cycle exercise did not differ from those measured during the Con. We previously reported that when a carbohydrate-protein drink was ingested either 5 or $30 \mathrm{~min}$ after high-intensity intermittent cycle exercise, GE was slower during the 5 min after exercise compared with the $30 \mathrm{~min} \mathrm{PE}$ and non-exercise trials ${ }^{(18)}$. In conscious rats, the residual rates in the stomach (i.e. the reciprocal index of GE rate) after ingestion of a liquid feed were evaluated at 0,10 or $20 \mathrm{~min}$ after high-intensity exercise ${ }^{(35)}$. When the feed was given at $0 \mathrm{~min}$ after exercise, the subsequent residual rates were the highest among the three trials ( 0 min: 72.8 (SD 5.9)\%, 10 min: 66.8 (SD 8.5) \%, 20 min: 38.2 (sD 3.7) \%). When the feed was ingested $20 \mathrm{~min}$ after exercise, the resultant GE rate was equivalent with that observed during the non-exercise trial (41.4 (SD 5.7)\%). Collectively, GE is delayed immediately after strenuous exercise and gradually recovers thereafter, irrespective of the exercise mode (i.e. between high-intensity intermittent cycle exercise in our previous study ${ }^{(18)}$ and following strenuous resistance exercise in the present study). Considering all these findings, we hope to further clarify the effects of exercise on GE, from both basic and mechanistic viewpoints. Until now, this subject was poorly understood.

The GE rate is modulated by several factors, including GI BF, $\mathrm{pH}$ and autonomic nervous activity ${ }^{(20)}$. We recently reported that decreased CA BF (i.e. hypoperfusion) following highintensity intermittent exercise acutely suppresses $\mathrm{GE}^{(18)}$. The CA BF supplies the stomach, pancreas, spleen and liver and supports digestive activities ${ }^{(36)}$. However, we did not measure GI BF (i.e. CA BF and SMA BF). It is well-known that reduced $\mathrm{BF}$ and/or vasoconstriction in GI structures can be elicited by prolonged leg cycling ${ }^{(9,10,14)}$ and static handgrip tasks (i.e. small muscle group exercises) ${ }^{(37)}$. However, there was no evidence of GI circulatory effects during and after whole-body resistance exercises. If GI hypoperfusion occurred during and/ or immediately after resistance exercises, the subsequent GE might be suppressed during the post-ingestion phase. In addition, GI reperfusion may also modulate the rate of GE. Experimental ischaemia-reperfusion of the CA acutely suppressed GE after food ingestion in rats ${ }^{(38)}$. This suppression occurred because of disruption of the interstitial cell Cajal network (i.e. pacemaker cells), which controls GI motility. Suppression also occurs secondary to neuronal NO synthase production, which is related to the regulation of gastric motility via gastric mucosal damage following acute ischaemia-reperfusion ${ }^{(38)}$. During the PE-5 condition, the acute suppression of GE may have been induced by GI hypoperfusion and/or reperfusion after strenuous exercise.

Silva et al. ${ }^{(35)}$ reported that, in rats, decreased blood $\mathrm{pH}$ immediately after strenuous exercise is associated with acute 

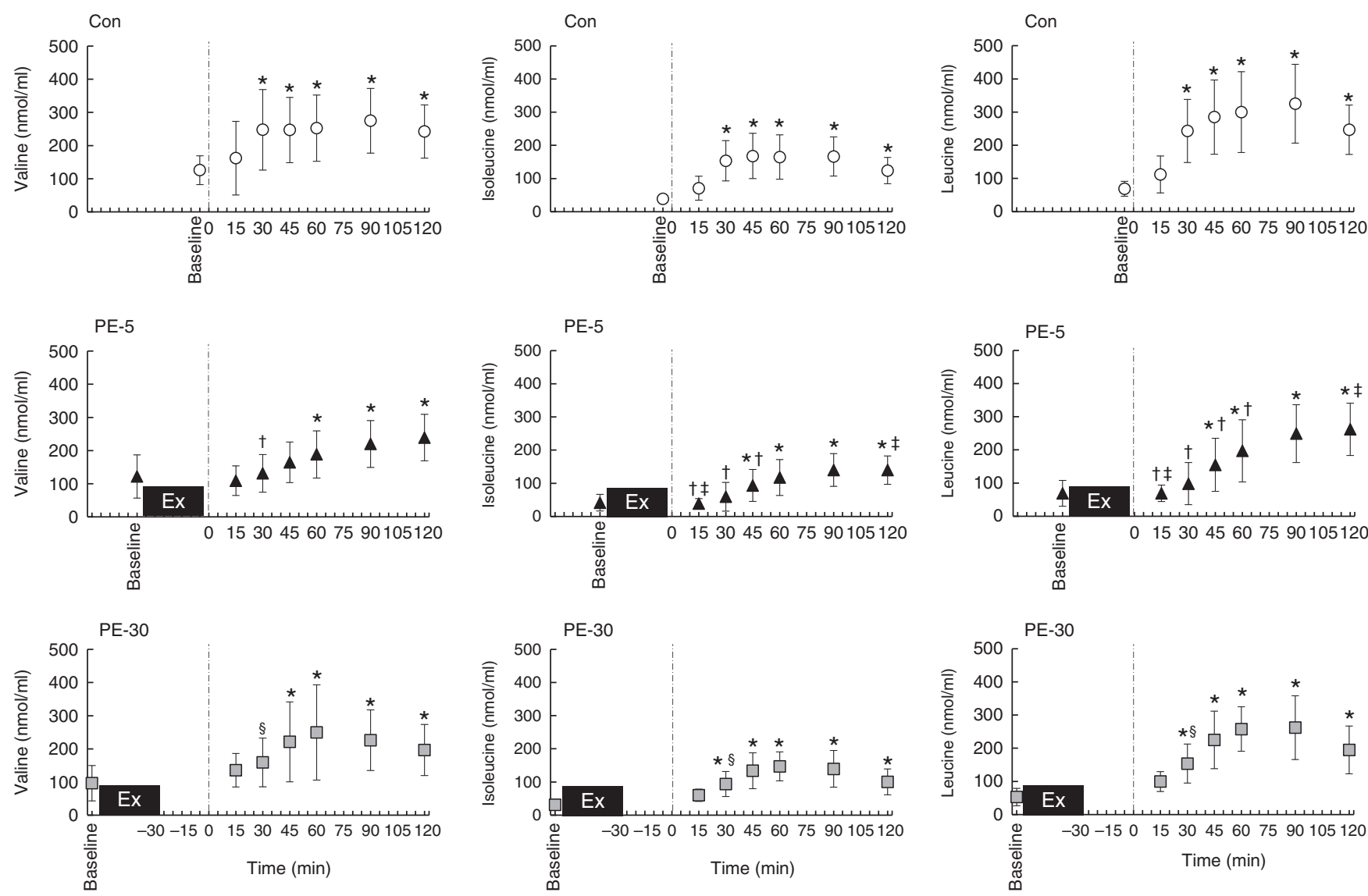

Fig. 5. Longitudinal plasma branched-chain amino acid (BCAA) response, except for those observed during exercise. The upper, middle and lower panels indicate no exercise (Con), 5 min post-exercise (PE-5) and 30 min post-exercise (PE-30), respectively. At 0 min, the vertical dotted line denotes the timing of carbohydrate-protein supplementation. Plasma BCAA responses were tested by two-way repeated ANOVA. When a significant difference was detected, we conducted Dunnett's and Tukey's post hoc tests to determine the effects of time (the change from baseline) and treatments, respectively. Values are means, with standard deviations represented by vertical bars. * Mean value was significantly different $(v$. baseline; $P<0.05)$; $†$ Mean value was significantly different for PE-5 $v$. Con $(P<0.05)$. $\ddagger$ Mean value was significantly different for PE-5 v. PE-30 $(P<0.05)$. § Mean value was significantly different for Con v. PE-30 v. Con $(P<0.05)$.

inhibition of GE. Immediately after exercise, blood lactate concentration was strongly correlated with residual rate for $15 \min ^{(35)}$. Interestingly, administration of sodium bicarbonate $40 \mathrm{~min}$ before exercise onset prevented reduction of blood $\mathrm{pH}$ levels immediately after exercise, accelerating $\mathrm{GE}^{(35)}$. Their results were comparable with our results in that blood lactate concentrations, just before ingestion of a nutrient-rich drink, with or without exercise, were significantly correlated with the rate of $\mathrm{GE}$ (i.e. $T_{\text {max-calc }}$ ).

The relative dominance of sympathetic/parasympathetic activity may also affect GE. In previous research, mental stress, pain and/or cold stimulation of the hand activates the sympathetic nervous system ${ }^{(39,40)}$, potentially suppressing $\mathrm{GE}^{(41,42)}$ and impairing gastric accommodation ${ }^{(43)}$. The PE-5 condition seemed to correspond to the highest level of sympathetic activation among the three trials. Here, we observed the highest HR values at $30 \mathrm{~min}$ after the ingestion of the nutrient-rich drink. Takarada et al. ${ }^{(44)}$ reported that immediately after resistance exercise with vascular occlusion, norepinephrine levels (which reflect sympathetic tone) peaked, returning to resting baseline levels $15 \mathrm{~min}$ after exercise. In addition, PE parasympathetic activation may partly regulate GE. Mild-to-moderate intensity exercise itself enhances subsequent gastric motility in healthy humans ${ }^{(45)}$ and rats ${ }^{(46)}$, and this enhanced response is absent in vagotomised rats ${ }^{(46)}$. In the present study, following ingestion of the nutrient-rich drink, greater HR was observed during the PE-5 condition than during either the PE-30 or Con condition. Thus, parasympathetic activation during the PE- 5 condition seemed to be lower than that observed during other trials. This was presumably related to acute GE suppression.

Increased blood glucose and plasma amino acid concentrations were slower to materialise during the PE- 5 condition than either the Con or PE-30 condition. This seemed to be influenced by differences in the rate of GE among the three trials. GE rate is a key factor for determining blood glucose excursion ${ }^{(47)}$ and SMA BF response ${ }^{(48)}$. Therefore, GE rate has been widely used as a measure of post-meal digestion and absorption. At $2 \mathrm{~h}$ after ingestion of a nutrient-rich drink, iAUC for BCAA were smaller during the PE-5 condition than during either the Con or PE-30 (nearing a significant difference) condition. This suggested impaired GI absorption during the PE-5 condition. van Wijck et $a l .{ }^{(17)}$ quantitatively evaluated dietary amino acids using a labelled protein and observed lower plasma dietary amino acid levels immediately after resistance exercise compared with a non-exercise trial. However, our results should be carefully interpreted based on the following points. 
Table 1. Participants' gastrointestinal symptoms and appetite scores§

(Mean values and standard deviations)

\begin{tabular}{|c|c|c|c|c|c|c|c|c|c|c|c|c|c|c|}
\hline \multirow[b]{2}{*}{ Trials } & \multicolumn{2}{|c|}{ Baseline } & \multicolumn{2}{|l|}{$A E-0$} & \multicolumn{2}{|l|}{$A E-25$} & \multicolumn{2}{|l|}{ Al-30 } & \multicolumn{2}{|l|}{$\mathrm{Al}-60$} & \multicolumn{2}{|l|}{ Al-90 } & \multicolumn{2}{|l|}{ Al-120 } \\
\hline & Mean & SD & Mean & SD & Mean & SD & Mean & SD & Mean & SD & Mean & SD & Mean & SD \\
\hline \multicolumn{15}{|c|}{ Stomach distension (mm) } \\
\hline Con & 5 & 9 & - & & - & & $34^{*}$ & 33 & 22 & 28 & 18 & 24 & 13 & 21 \\
\hline PE-5 & 4 & 7 & 24 & 30 & - & & $44^{*}$ & 33 & $30^{\star}$ & 27 & 18 & 24 & 13 & 18 \\
\hline PE-30 & 3 & 6 & $27^{\star}$ & 34 & 20 & 26 & $38^{\star}$ & 33 & $25^{\star}$ & 26 & 16 & 16 & 12 & 16 \\
\hline \multicolumn{15}{|l|}{ Stomach pain $(\mathrm{mm})$} \\
\hline Con & 0 & 1 & - & & - & & 0 & 0 & 0 & 0 & 0 & 0 & 0 & 0 \\
\hline PE-5 & 0 & 0 & 2 & 8 & - & & 1 & 2 & 0 & 0 & 0 & 0 & 0 & 0 \\
\hline PE-30 & 0 & 0 & $9^{*}$ & 18 & 0 & 0 & 0 & 0 & 0 & 0 & 0 & 0 & 0 & 0 \\
\hline \multicolumn{15}{|l|}{ Nausea (mm) } \\
\hline Con & 0 & 0 & - & & - & & 0 & 0 & 0 & 1 & 0 & 0 & 0 & 1 \\
\hline PE-5 & 0 & 0 & $12^{*}$ & 24 & - & & $8 \dagger$ & 10 & 7 & 14 & 0 & 0 & 0 & 0 \\
\hline PE-30 & 0 & 1 & $19^{*}$ & 31 & 9 & 16 & 2 & 6 & 1 & 2 & 1 & 3 & 0 & 1 \\
\hline \multicolumn{15}{|l|}{ Desire to eat $(\mathrm{mm})$} \\
\hline Con & 66 & 23 & - & & - & & 52 & 27 & 54 & 21 & 67 & 17 & 78 & 13 \\
\hline PE-5 & 72 & 23 & $18^{\star}$ & 21 & - & & $28^{*} \dagger \ddagger$ & 21 & $45^{*}$ & 20 & 58 & 22 & 69 & 25 \\
\hline PE-30 & 76 & 21 & $15^{*}$ & 12 & $38^{*}$ & 18 & $46^{*}$ & 20 & $55^{*}$ & 12 & 61 & 14 & 73 & 13 \\
\hline \multicolumn{15}{|l|}{ Hunger (mm) } \\
\hline Con & 72 & 24 & - & & - & & $37^{*}$ & 25 & $50^{*}$ & 21 & 60 & 24 & 75 & 23 \\
\hline PE-5 & 75 & 17 & $19^{*}$ & 14 & - & & $19^{*} \dagger \ddagger$ & 17 & $47^{*}$ & 18 & 62 & 21 & 68 & 24 \\
\hline PE-30 & 81 & 19 & $20^{*}$ & 18 & $37^{\star}$ & 31 & $35^{\star}$ & 20 & $52^{*}$ & 16 & $57^{*}$ & 18 & 77 & 10 \\
\hline \multicolumn{15}{|l|}{ Fullness (mm) } \\
\hline Con & 10 & 11 & - & & - & & $44^{*}$ & 30 & $38^{*}$ & 25 & 23 & 23 & 16 & 22 \\
\hline PE-5 & 12 & 13 & 24 & 22 & - & & $61^{*}$ & 26 & $39^{*}$ & 25 & 30 & 24 & 18 & 24 \\
\hline PE-30 & 7 & 9 & $33^{*}$ & 30 & $31^{*}$ & 25 & $50^{*}$ & 30 & $35^{*}$ & 23 & 23 & 16 & 13 & 15 \\
\hline \multicolumn{15}{|c|}{ Prospective consumption (mm) } \\
\hline Con & 77 & 15 & - & & - & & $57^{\star}$ & 17 & $61^{*}$ & 16 & 69 & 21 & 77 & 18 \\
\hline PE-5 & 78 & 16 & $21^{*}$ & 23 & - & & $28^{*} \dagger \ddagger$ & 18 & $51^{*}$ & 17 & 64 & 18 & 66 & 27 \\
\hline PE-30 & 82 & 19 & $16^{*}$ & 16 & $38^{*}$ & 20 & $46^{*}$ & 19 & $56^{*}$ & 15 & 68 & 15 & 78 & 14 \\
\hline \multicolumn{15}{|c|}{ Average appetite score $(\mathrm{mm})$} \\
\hline Con & 77 & 16 & - & & - & & $51^{*}$ & 21 & $57^{\star}$ & 18 & 68 & 19 & 78 & 18 \\
\hline PE-5 & 78 & 15 & $33^{*}$ & 11 & - & & $29^{*} \dagger \ddagger$ & 17 & $51^{*}$ & 17 & 63 & 20 & 71 & 23 \\
\hline PE-30 & 83 & 15 & $29^{*}$ & 13 & $45^{\star}$ & 19 & $44^{*}$ & 17 & $57^{*}$ & 14 & $66^{*}$ & 12 & 79 & 11 \\
\hline
\end{tabular}

$\mathrm{AE}$, after exercise; $\mathrm{Al}$, after ingestion (of nutrient drink); Con, control trial; $\mathrm{PE}$, post-exercise.

* Mean value was significantly different from that of baseline $(P<0.05)$.

† Mean value was significantly different for PE-5 $v$. Con $(P<0.05)$

$\ddagger$ Mean value was significantly different for PE-5 v. PE-30 $(P<0.05)$.

$\S$ Participants' gastrointestinal symptoms and appetite scores were tested using a two-way repeated ANOVA. When a significant difference was detected, Dunnett's and Tukey's post hoc tests were conducted to determine the effects of time (the change from baseline) and treatments, respectively.

First, our results reflect the appearance of amino acids into the blood circulation that were derived from both dietary protein and from muscle protein breakdown and uptake ${ }^{(2)}$. Therefore, the plasma amino acid responses did not precisely reflect the absolute amount of pure dietary protein absorbed from the small intestine. Second, the time following ingestion of the nutrient-rich drink was relatively short. Specifically, during the PE- 5 condition, some participants seemed not to attain peak plasma amino acid response levels. The plasma amino acid response should be measured until it returns to baseline levels. Therefore, future studies should evaluate dietary amino acid kinetics in blood circulation and its uptake into skeletal muscle using a protocol similar to this study.

During the PE- 5 trial, the timing of nutrient-rich drink after strenuous resistance exercise was faster for $25 \mathrm{~min}$ than in PE-30, and then the PE-5 condition was associated with slower GE (e.g. $T_{\text {max-calc }}$ ) for 21 min compared with PE-30. According to these results, the effects of timing of nutrient-rich drink ingestion on GE, after strenuous resistance exercise, might not attribute substantive meaning to the subsequent digestive and absorptive rates. However, even if it does, to minimise the magnitude of PE GI distress and anorexia in athletes and training enthusiasts, nutrient ingestion at PE-5 (i.e. immediately after exercise) might be not recommended. The present data provide useful information for preventing GI distress and encouraging effective physical fatigue recovery in athletes. In summary, the timing of dietary supplementation after highintensity resistance exercise impacts GE, blood glucose, plasma BCAA responses and GI symptoms. Our findings may have implications for future research into the timing of dietary supplementation after exercise.

\section{Acknowledgements}

The authors thank the participants for their involvement in this study. 
This study was supported by the Japan Society for the Promotion of Science KAKENHI (grant no. 40714746 to H. K. and grant no. $17 \mathrm{~K} 01616$ to Y. F.)

H. K. and Y. F. conceptualised and designed the study, generated, collected, assembled, analysed and interpreted study data and drafted the revised manuscript. K. S., K. T., R. K., S. T. and T. K. assisted with the collection, assembly, analysis and interpretation of data. M. Y. E. and A. M. assisted with the interpretation of data.

The authors declare that there are no conflicts of interest.

\section{References}

1. Thomas DT, Erdman KA \& Burke LM (2016) Position of the Academy of Nutrition and Dietetics, Dietitians of Canada, and the American College of Sports Medicine: nutrition and athletic performance. $J$ Acad Nutr Diet 116, 501-528.

2. Levenhagen DK, Gresham JD, Carlson MG, et al. (2001) Postexercise nutrient intake timing in humans is critical to recovery of leg glucose and protein homeostasis. Am J Physiol Endocrinol Metab 280, E982-E993.

3. Ivy JL \& Ferguson-Stegall LM (2014) Nutrient timing: the means to improved exercise performance, recovery, and training adaptation. Am J Lifestyle Med 8, 246-259.

4. Peters HP, Bos M, Seebregts L, et al. (1999) Gastrointestinal symptoms in long-distance runners, cyclists, and triathletes: prevalence, medication, and etiology. Am J Gastroenterol 94, $1570-1581$.

5. ter Steege RW, Van der Palen J \& Kolkman JJ (2008) Prevalence of gastrointestinal complaints in runners competing in a long-distance run: an internet-based observational study in 1281 subjects. Scand J Gastroenterol 43, $1477-1482$.

6. van Wijck K, Lenaerts K, Grootjans J, et al. (2012) Physiology and pathophysiology of splanchnic hypoperfusion and intestinal injury during exercise: strategies for evaluation and prevention. Am J Physiol Gastrointest Liver Physiol 303, G155-G168.

7. de Oliveira EP, Burini RC \& Jeukendrup A (2014) Gastrointestinal complaints during exercise: prevalence, etiology, and nutritional recommendations. Sports Med 44, Suppl. 1, S79-S85.

8. ter Steege RW \& Kolkman JJ (2012) Review article: the pathophysiology and management of gastrointestinal symptoms during physical exercise, and the role of splanchnic blood flow. Aliment Pharmacol Ther 35, 516-528.

9. Perko MJ, Nielsen HB, Skak C, et al. (1998) Mesenteric, coeliac and splanchnic blood flow in humans during exercise. J Physiol 513, 907-913.

10. Qamar MI \& Read AE (1987) Effects of exercise on mesenteric blood flow in man. Gut 28, 583-587.

11. Otte JA, Oostveen E, Geelkerken RH, et al. (2001) Exercise induces gastric ischaemia in healthy volunteers: a tonometry study. J Appl Physiol 91, 866-871.

12. ter Steege RW, Geelkerken RH, Huisman AB, et al. (2012) Abdominal symptoms during physical exercise and the role of gastrointestinal ischaemia: a study in 12 symptomatic athletes. Br J Sports Med 46, 931-935.

13. van Wijck K, Lenaerts K, van Loon LJ, et al. (2011) Exerciseinduced splanchnic hypoperfusion results in gut dysfunction in healthy men. PLOS ONE 6, e22366.

14. Rehrer NJ, Smets A, Reynaert H, et al. (2001) Effect of exercise on portal vein blood flow in man. Med Sci Sports Exerc 33, 1533-1537.
15. Osada T, Iwane H, Katsumura T, et al. (2012) Relationship between reduced lower abdominal blood flows and heart rate in recovery following cycling exercise. Acta Physiol 204, 344-353.

16. Rowell LB, Blackmon JR, Martin RH, et al. (1965) Hepatic clearance of indocyanine green in man under thermal and exercise stresses. J Appl Physiol 20, 384-394.

17. van Wijck K, Pennings B, van Bijnen AA, et al. (2013) Dietary protein digestion and absorption are impaired during acute postexercise recovery in young men. Am J Physiol Regul Integr Comp Physiol 304, R356-R361.

18. Kashima H, Harada N, Miyamoto K, et al. (2017) Timing of post-exercise carbohydrate-protein supplementation: roles of gastrointestinal blood flow and mucosal cell damage on gastric emptying in humans. J Appl Physiol 123, 606-613.

19. Bi L \& Triadafilopoulos G (2003) Exercise and gastrointestinal function and disease: an evidence-based review of risks and benefits. Clin Gastroenterol Hepatol 1, 345-355.

20. Horner KM, Schubert MM, Desbrow B, et al. (2015) Acute exercise and gastric emptying: a meta-analysis and implications for appetite control. Sports Med 45, 659-678.

21. Leiper JB (2015) Fate of ingested fluids: factors affecting gastric emptying and intestinal absorption of beverages in humans. Nutr Rev 2, 57-72.

22. Murray R (1987) The effects of consuming carbohydrateelectrolyte beverages on gastric emptying and fluid absorption during and following exercise. Sports Med 4, 322-351.

23. Evans GH, Watson P, Shirreffs SM, et al. (2016) The effect of exercise intensity on subsequent gastric emptying rate in humans. Int J Sport Nutr Exerc Metab 26, 128-134.

24. Morton RW, McGlory C \& Phillips SM (2015) Nutritional interventions to augment resistance training-induced skeletal muscle hypertrophy. Front Physiol 6, 245.

25. Evans GH, James LJ, Shirreffs SM, et al. (2017) Optimizing the restoration and maintenance of fluid balance after exerciseinduced dehydration. J Appl Physiol 122, 945-951.

26. Brennan IM, Feltrin KL, Nair NS, et al. (2009) Effects of the phases of the menstrual cycle on gastric emptying, glycemia, plasma GLP-1 and insulin, and energy intake in healthy lean women. Am J Physiol Gastrointest Liver Physiol 297, G602-G610.

27. Ghoos YF, Maes BD, Geypens BJ, et al. (1993) Measurement of gastric emptying rate of solids by means of a carbonlabeled octanoic acid breath test. Gastroenterology 104, 1640-1647.

28. Haycock GB, Schwartz GJ, Wisotsky DH, et al. (1978) Geometric method for measuring body surface area: a heightweight formula validated in infants, children, and adults. J Pediatr 93, 62-66.

29. Braden B, Adams S, Duan LP, et al. (1995) The $\left[{ }^{13} C\right]$ acetate breath test accurately reflects gastric emptying of liquids in both liquid and semisolid test meals. Gastroenterology $\mathbf{1 0 8}$, 1048-1055.

30. Chew CG, Bartholomeusz FD, Bellon M, et al. (2003) Simultaneous ${ }^{13} \mathrm{C} /{ }^{14} \mathrm{C}$ dual isotope breath test measurement of gastric emptying of solid and liquid in normal subjects and patients: comparison with scintigraphy. Nucl Med Rev Cent East Eur 6, 29-33.

31. Sanaka M, Yamamoto T, Nakayama S, et al. (2008) Reliability of the time to maximal $\left[{ }^{13} \mathrm{CO}_{2}\right]$ excretion and the half- $\left[{ }^{13} \mathrm{CO}_{2}\right]$ excretion time as a gastric emptying parameter: assessments using the Wagner-Nelson method. J Smooth Muscle Res $\mathbf{4 3}$, 201-209.

32. Abdulrazzaq YM \& Ibrahim A (2001) Determination of amino acids by ion-exchange chromatography on filter paper spotted blood samples stored at different temperatures and for 
different periods: comparison with capillary and venous blood. Clin Biochem 34, 399-406.

33. Anderson GH, Catherine NL, Woodend DM, et al. (2002) Inverse association between the effect of carbohydrates on blood glucose and subsequent short-term food intake in young men. Am J Clin Nutr 76, 1023-1030.

34. Samra RA \& Anderson GH (2007) Insoluble cereal fibre reduces appetite and short-term food intake and glycemic response to food consumed $75 \mathrm{~min}$ later by healthy men. Am J Clin Nutr 86, 972-979.

35. Silva MT, Palheta-Junior RC, Sousa DF, et al. (2014) Sodium bicarbonate treatment prevents gastric emptying delay caused by acute exercise in awake rats. J Appl Physiol 116, 1133-1141.

36. Granger DN, Holm L \& Kvietys P (2015) The gastrointestinal circulation: physiology and pathophysiology. Compr Physiol 5, 1541-1583.

37. Waaler BA, Toska K \& Eriksen M (1999) Involvement of the human splanchnic circulation in pressor response induced by handgrip contraction. Acta Physiol Scand 166, 131-136.

38. Suzuki S, Suzuki H, Horiguchi K, et al. (2010) Delayed gastric emptying and disruption of the interstitial cells of Cajal network after gastric ischaemia and reperfusion. Neurogastroenterol Motil 22, 585-593.

39. Herd JA (1991) Cardiovascular response to stress. Physiol Rev 71, 305-330.

40. Victor RG, Leimbach WN Jr, Seals DR, et al. (1987) Effects of the cold pressor test on muscle sympathetic nerve activity in humans. Hypertension 9, 429-436.
41. Fone DR, Horowitz M, Maddox A, et al. (1990) Gastroduodenal motility during the delayed gastric emptying induced by cold stress. Gastroenterology 98, 1155-1161.

42. Roland J, Dobbeleir A, Vandevivere J, et al. (1990) Effect of mild mental stress on solid phase gastric emptying in healthy subjects. Nucl Med Commun 11, 319-326.

43. Geeraerts B, Vandenberghe J, Van Oudenhove L, et al. (2005) Influence of experimentally induced anxiety on gastric sensorimotor function in humans. Gastroenterology 129, $1437-1444$.

44. Takarada Y, Nakamura Y, Aruga S, et al. (2000) Rapid increase in plasma growth hormone after low-intensity resistance exercise with vascular occlusion. J Appl Physiol 88, 61-65.

45. Kato M, Sakai T, Yabe K, et al. (2004) Gastric myoelectrical activity increases after moderate-intensity exercise with no meals under suppressed vagal nerve activity. Jpn J Physiol 54, 221-228.

46. Wang Y, Kondo T, Suzukamo Y, et al. (2010) Vagal nerve regulation is essential for the increase in gastric motility in response to mild exercise. Tohoku J Exp Med 222, 155-163.

47. Marathe CS, Rayner CK, Jones KL, et al. (2013) Relationships between gastric emptying, postprandial glycemia, and incretin hormones. Diabetes Care 36, 1396-1405.

48. Sidery MB, Macdonald IA \& Blackshaw PE (1994) Superior mesenteric artery blood flow and gastric emptying in humans and the differential effects of high fat and high carbohydrate meals. Gut 35, 186-190. 